
Modulates $\mathrm{Ca}^{2+}$-Mediated Smooth

SUBJECT AREAS:

PHARMACOLOGY

PHYSIOLOGY

BIOPHYSICS

MEDICAL RESEARCH

Received

25 September 2012

Accepted

20 November 2012

Published

14 December 2012

Correspondence and requests for materials should be addressed to S.N. (h44673a@nucc. cc.nagoya-u.ac.jp)

\section{Muscle Contraction}

Shunichi Kajioka' , Fumi Takahashi-Yanaga ${ }^{2}$, Nouval Shahab' ${ }^{1}$ Mitsuho Onimaru ${ }^{3}$, Miho Matsuda 5 , Ryosuke Takahashi' ${ }^{1}$ Haruhiko Asano ${ }^{7}$, Hiromitsu Morita ${ }^{6}$, Sachio Morimoto ${ }^{2}$, Yoshikazu Yonemitsu ${ }^{4}$, Maya Hayashi' ${ }^{1}$ Narihito Seki' ${ }^{1}$, Toshiuyki Sasaguri ${ }^{2}$, Masato Hirata ${ }^{5}$, Shinsuke Nakayama ${ }^{8}$ \& Seiji Naito'

${ }^{1}$ Department of Urology, ${ }^{2}$ Department of Clinical Pharmacology, ${ }^{3}$ Department of Pathology, ${ }^{4}$ Operating Unit for Clinical Trials of Gene Therapy, Graduate School of Medical Sciences, ${ }^{5}$ Laboratory of Molecular \& Cellular Biochemistry, Faculty of Dental Science, Kyushu University, ${ }^{6}$ Special Patient Oral Care Unit, Kyushu University Hospital Fukuoka 812-8582, Japan, ${ }^{7}$ Department of Biomedical Sciences, College of Life and Health Sciences, Chubu University, Kasugai Aichi, 487-8501, Japan, ${ }^{8}$ Department of Cell Physiology, Nagoya University Graduate School of Medicine, Nagoya 466-8550, Japan.

Mechanisms linked to actin filaments have long been thought to cooperate in smooth muscle contraction, although key molecules were unclear. We show evidence that cardiac troponin $\mathrm{T}(\mathrm{cTnT})$ substantially contributes to $\mathrm{Ca}^{2+}$-mediated contraction in a physiological range of cytosolic $\mathrm{Ca}^{2+}$ concentration $\left(\left[\mathrm{Ca}^{2+}\right]_{\mathrm{i}}\right)$. cTnT was detected in various smooth muscles of the aorta, trachea, gut and urinary bladder, including in humans. Also, cTnT was distributed along with tropomyosin in smooth muscle cells, suggesting that these proteins are ready to cause smooth muscle contraction. In chemically permeabilised smooth muscle of $\mathrm{cTnT}^{+/-}$mice in which $\mathrm{cTnT}$ reduced to $\sim 50 \%$, the $\mathrm{Ca}^{2+}$-force relationship was shifted toward greater $\left[\mathrm{Ca}^{2+}\right]_{\mathrm{i}}$, indicating a sizeable contribution of cTnT to smooth muscle contraction at $\left[\mathrm{Ca}^{2+}\right]_{\mathrm{i}}<1 \mu \mathrm{M}$. Furthermore, addition of supplemental $\mathrm{TnI}$ and $\mathrm{TnC}$ reconstructed a troponin system to enhance contraction. The results indicated that a $\mathrm{Tn} / \mathrm{Tn}$-like system on actin-filaments cooperates together with the thick-filament pathway.

ncreases in cytosolic $\mathrm{Ca}^{2+}$ concentration $\left(\left[\mathrm{Ca}^{2+}\right]_{\mathrm{i}}\right)$ cause contraction in any muscle. It is, however, believed that regulatory mechanisms underlying the $\mathrm{Ca}^{2+}$-mediated actin-myosin interaction differ fundamentally between muscle types. Namely, in striated skeletal and cardiac muscles, $\mathrm{Ca}^{2+}$ bound to troponin (Tn) on actin filaments removes the inhibitory effect of Tn on the actin-myosin interaction ${ }^{1,2}$. In contrast, smooth muscles employ the $\mathrm{Ca}^{2+} /$ calmodulin complex to activate myosin light chain kinase (MLCK), in turn resulting in an actin-myosin interaction through phosphorylation of the myosin light chain (MLC) $)^{3-5}$.

Although a major contribution of myosin thick-filaments to smooth muscle contraction is widely accepted, there is considerable evidence that thin filament-linked mechanisms are operating in parallel (e.g. contribution of caldesmon and calponin $)^{6-10}$. In addition, a number of chemicals, e.g. activators for protein-kinase C and PI3kinase $^{11-14}$, are known to cause contraction independent of MLC phosphorylation. Despite this, to date, the functional contribution of a troponin-regulatory system is ambiguous.

In the present study, we show evidence that cardiac troponin $\mathrm{T}(\mathrm{cTnT})$ exists in smooth muscle, including humans, and substantially contributes to $\mathrm{Ca}^{2+}$-mediated contraction in a physiological range of $\left[\mathrm{Ca}^{2+}\right]_{\mathrm{i}}$ especially below $1 \mu \mathrm{M}$. The results indicate that a Tn-like system on actin-filaments cooperates together with the myosin regulatory pathway in smooth muscle, as the phosphorylation of myosin light chain modulates contractions in striated muscles ${ }^{15}$. The present findings on TnT suggest new insight into medical treatments to overcome numerous diseases related to the malfunction of smooth muscle distributed over the body: The Tn-like system in smooth muscle is a possible target in pharmacological and genetic therapies. In addition, mutations on TnT and associated proteins are likely to also alter smooth muscle contractility through the Tn-like system, as observed in familial myopathies of cardiac and skeletal muscles ${ }^{2,16}$.

\section{Results}

Expression of troponins in smooth muscle. In striated muscle, a complex of three troponins, namely $\mathrm{Tn} T / \mathrm{TnC} /$ TnI, acts as a molecular switch of the actin-myosin interaction ${ }^{2}$. Thus, we first explored the expression of cardiac 


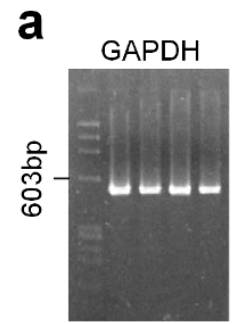

1234
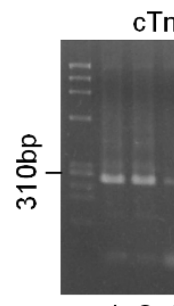

TnT cTnl

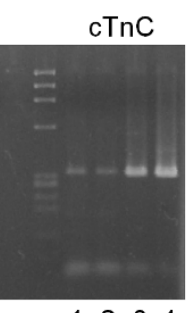

1234

1234

1 HUB-1 (P1)

2 HUB-2 (P2)

3 HUB-3 (C)

$4 \mathrm{MH}$

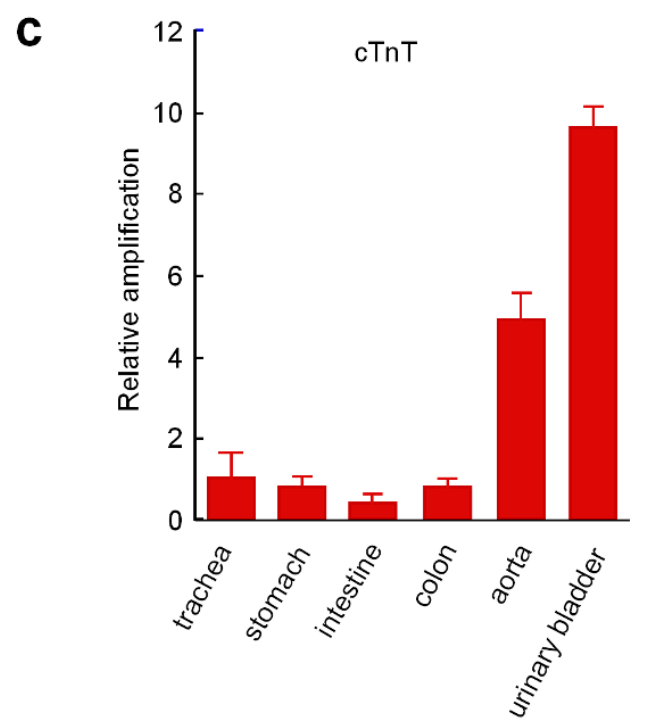

b

b $\quad$ TnT

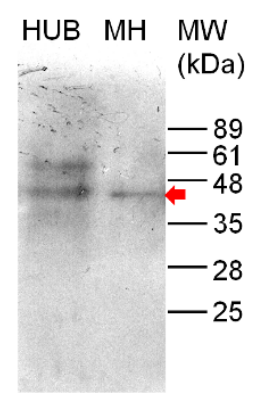

cTnl

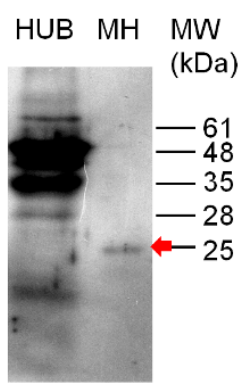

cTnC HUB MH MW

(kDa)

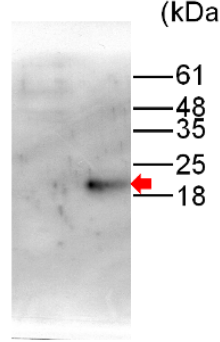

d

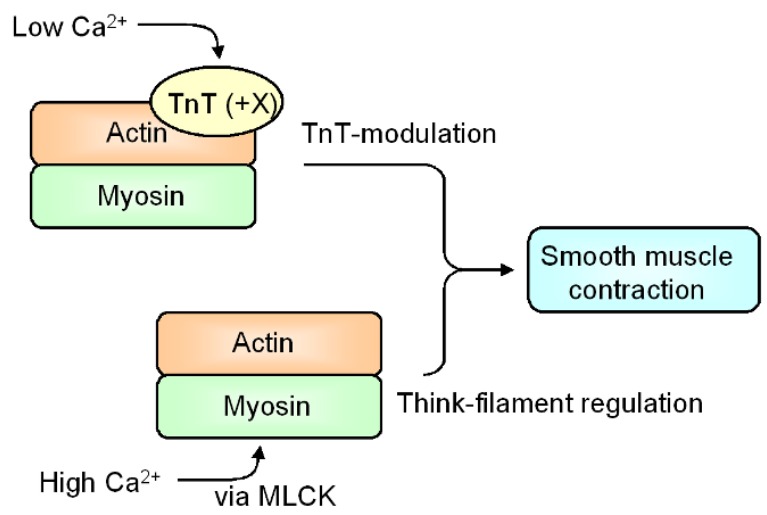

Figure 1 Expression of cardiac troponins in smooth muscle. (a) RT-PCR detection of cardiac troponins (cTnT/cTnI/cTnC) in detrusor smooth muscle. Specimens used were from human urinary bladder (HUB) detrusor smooth muscle (cystectomy patients: P-1 and P-2), commercial tRNA of human urinary bladder smooth muscle (C), and mouse cardiac ventricular muscle (MH). (b) Western blot examination of cardiac troponins in human detrusor smooth muscle and mouse heart (MH). Red arrow indicates the expected molecular weight (MW) of each cardiac troponin. (c) Real-time PCR quantification of cTnT in commercial tRNA of various human smooth muscle tissues. cTnT expression is plotted relative to that in trachea. (d) Schematic diagram. cTnT may contribute to smooth muscle contraction by modulating actin filaments.

troponins (cTn) in detrusor smooth muscle isolated from the urinary bladder of humans. RT-PCR detected all three components of cTn (Fig. 1a). Although amplicons were faint in some of cTnI and cTnT examinations, the sequences of the amplicons were exactly the same as known sequences of cDNAs of cTnT, cTnI and cTnC in human detrusor, respectively (Fig. S1). Whilst immunoblotting clearly detected only cTnT (Fig. 1b). Positive controls were obtained from mouse heart $(\mathrm{MH})$.

To examine the tissue-dependent expression of cTn among smooth muscles, real-time PCR was carried out using commercial standard mRNA samples obtained from the human trachea, stomach, small intestine, colon, aorta and urinary bladder. The amount of cTnT varied among smooth muscles. Urinary bladder (detrusor) contained 9.5 times more than trachea, while gastrointestinal smooth muscles contained only 35-77\% (Fig. 1c). The amounts of cTnC and cTnI also varied among tissues (Fig. S2). These results imply that a part of contraction is mediated via the Tn complex and/or a Tn-like complex, including replaceable proteins on actin filaments along with a major control of myosin filament activation (Fig. 1d), and that detrusor smooth muscle is a suitable tissue to demonstrate cTnT-related contraction (Fig. 1c).
Colocalisation of TnT with tropomyosin. The Tn complex promotes an actin-myosin interaction by shifting tropomyosin, which covers myosin-binding sites on actin filaments in striated muscle. On the other hand, smooth muscle is known to contain tropomyosin in the cytosplasm, although its physiological function is not well understood so far ${ }^{17,18}$. Thus, we assessed if $\mathrm{TnT}$ and tropomyosin colocalize in the cytoplasm of smooth muscle cells. In a pair of thin sections prepared from human detrusor, cTnT fluorescence was observed in smooth muscle bundles, but not in the connective tissue region except for the wall of veins (Fig. 2a, 2b).

To rule out background fluorescence from cells other than smooth muscle, cultured detrusor smooth muscle cells were next used. These cells were clearly stained with anti-cTnT (Fig. 2c, 2e) or anti-tropomyosin antibodies (Fig. 2f, 2h): double staining with cellular nuclei (Fig. 2d, 2e and Fig. 2g, 2h). Also, the overlap of cTnT and tropomyosin fluorescence is shown expanded: triple staining (Fig. 2i-2l). Furthermore, RT-PCR detected both cTnT and MYL6 (smooth muscle indicator) in the same cultured cells prepared from the human detrusor (Fig. $2 \mathrm{~m}$ ). These results suggest that cTnT and tropomyosin are fixed together and are ready to cause smooth muscle contraction if any signal targets thin filaments to be activated. 

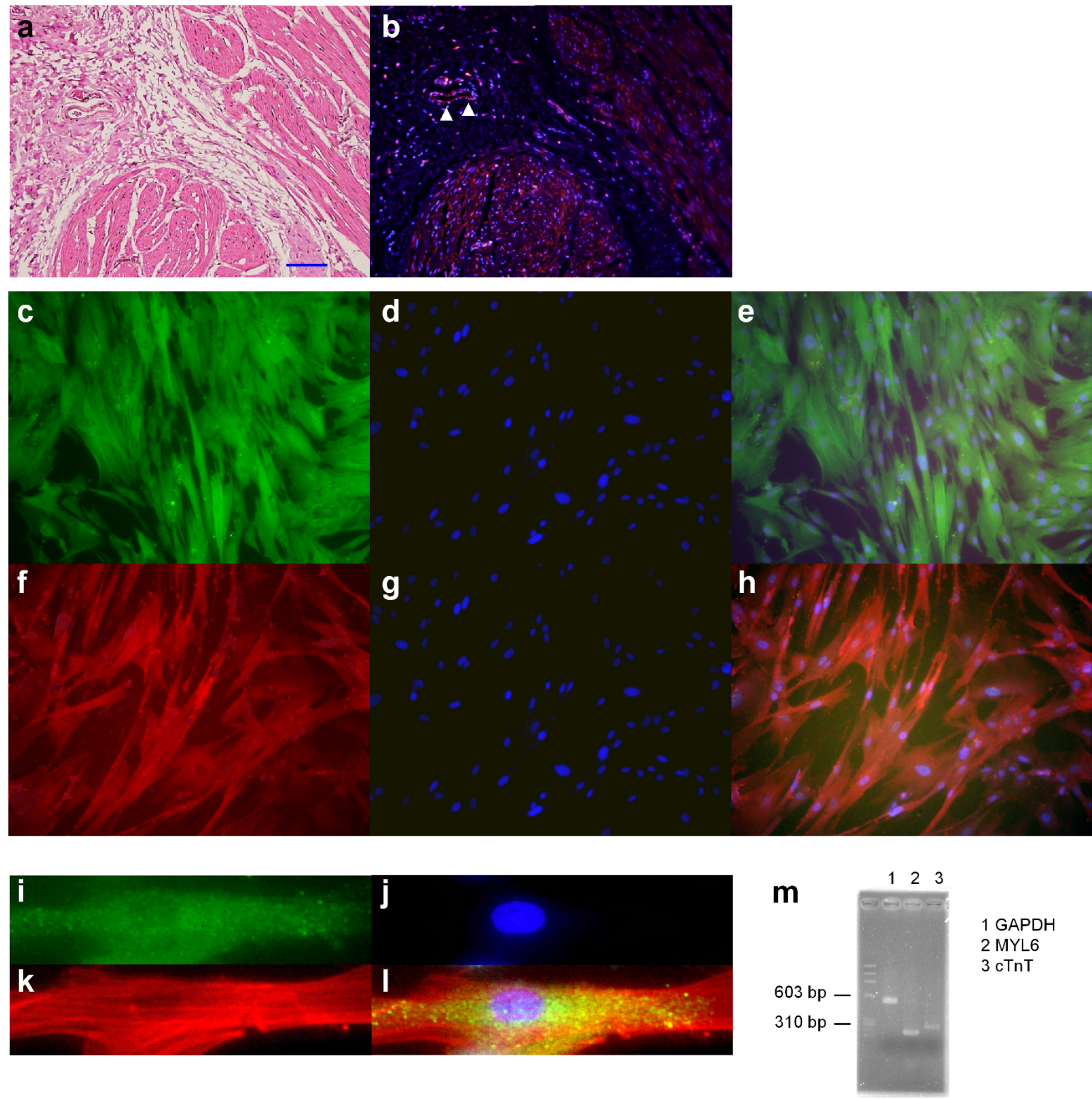

Figure $2 \mid$ Histological analysis. (a and b) A pair of thin sections of human urinary bladder detrusor smooth muscle were used. Hematoxylin-eosin (HE) staining in (a), and fluorescence of cTnT (red) and nuclear (blue) in (b). The scale bar indicates $150 \mu \mathrm{m}$ (a), (c-l) Cultured smooth muscle cells of human detrusor smooth muscle were used in fluorescence photomicrographs. (c-e) Double staining of cTnT (green, c) and nuclear (blue, d); merge (e). (f-h) Double staining of tropomyosin (TM) (red, f) and nuclear (blue, g); merge (h). (i-l) Triple staining of cTnT (green, i), nuclear (blue, j) and tropomyosin (TM) (red, k) are shown expanded; merge (l). (m) The expression of GAPDH, MYL6 (smooth muscle marker) and cTnT in the same cultured smooth muscle cells was examined using RT-PCR.

$\mathrm{Ca}^{2+}$-mediated contraction. In order to assess whether $\left[\mathrm{Ca}^{2+}\right]_{\mathrm{i}^{-}}$ dependent force development employs endogenous TnT on tropomyosin to induce contraction, we permeabilized human detrusor strips with $\beta$-escin to control $\left[\mathrm{Ca}^{2+}\right]_{\mathrm{i}}$. After chemical permeabilization at least three subsequent applications of $1 \mu \mathrm{M} \mathrm{Ca}^{2+}$ caused stable contractions of similar amplitude (i, ii and iii in Fig. 3a): $0.97 \pm 0.04$ and $0.94 \pm 0.06$ in the second and third contraction, respectively relative to the first one $(n=5)$.

cTnT appears to be fixed on tropomyosin in the cytoplasm (Fig. 2c-2f), while cTnI and cTnC are less than cTnT (Fig. 1c), and the peameabilization procedure is likely to release these troponin proteins along with replaceable proteins, if any, toward the extracellular space. We thus examined the effects of exogenous cTnI and cTnC. The application of cTnI after first contraction decreased the amplitude of the third contraction to $0.55 \pm 0.04(n=6$; Fig. $3 b)$, whereas the application of $\mathrm{cTnC}$ restored it to $1.23 \pm 0.03(\mathrm{n}=6$; Fig. 3c). The amplitude of the third contraction is summarized in Fig. 3d (see also Fig. S3 showing consistent effects of cTnI and cTnC irrespective of exposure time). An immunoblot shows the existence of cTnT after the permeabilization procedure (Fig. 3e: lane 1 in 
a

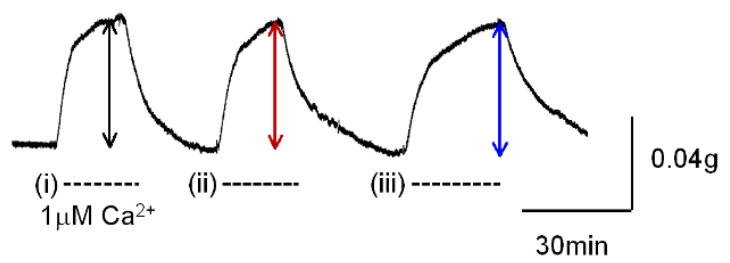

b

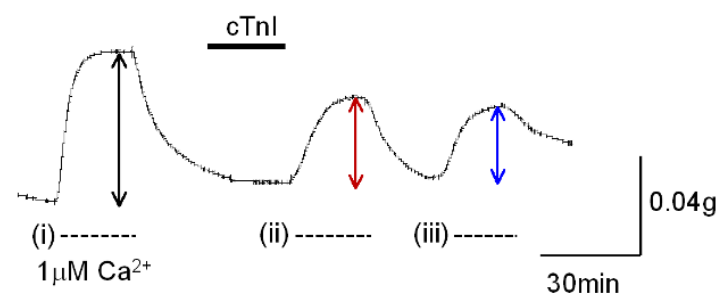

C

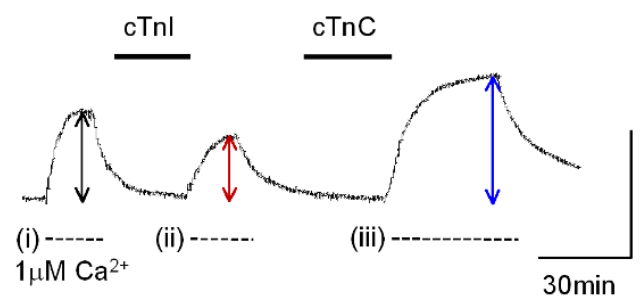

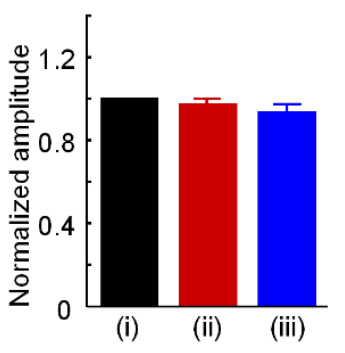
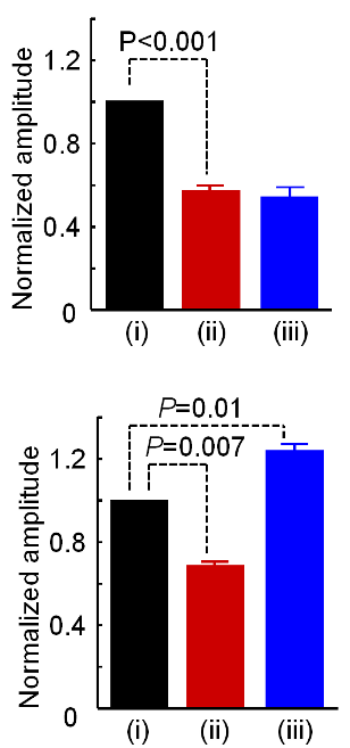
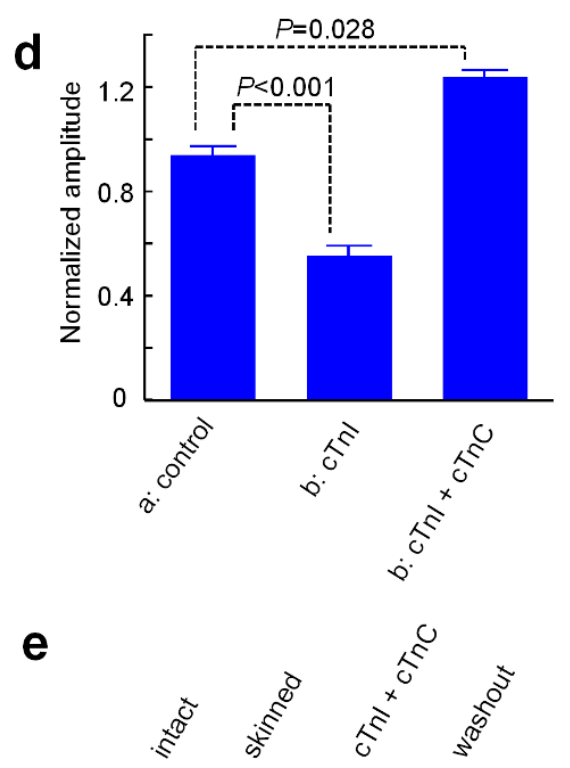

cTnT

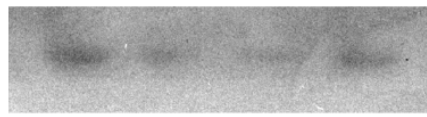

cTnl

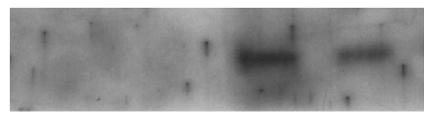

cTnC

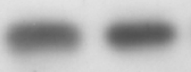

Figure 3 Potentiation of $\mathrm{Ca}^{2+}$-dependent force development by constituting $\mathrm{Tn}$ complex. Three contractions (i, ii and iii) were successively induced by applying a bathing solution containing $1 \mu \mathrm{M}\left[\mathrm{Ca}^{2+}\right]$ in chemically permeabilised strips prepared from human detrusor smooth muscle. (a) Left panel, an example trace showing a measurement of forcedevelopment; right panel, summary graph for the three successive contractions. cTnI was added to the bathing solution before the second contraction in $\mathrm{b}$, and $\mathrm{cTnC}$ was additionally applied before the third contraction in $\mathrm{c}$. The amplitude of the third contraction relative to the first one is plotted in $\mathrm{d}$. The application of cTnI reduced, and the application of both cTnI and cTnC potentiated the third contraction statistically significantly ( ${ }^{*} P<0.05$ and ${ }^{* *} P<0.01$, respectively, unpaired $t$-test). (e) Western blot examinations of Tn proteins. Lane 1 and lane 2 show before and after permeabilisation, respectively. Lane 3 and 4 correspond to the force measurement $\mathrm{c}$ before and after washout with relaxing solution for $30 \mathrm{~min}$, respectively.

control; lane 2 after permeabilization). Also, after the application of exogenous $\mathrm{cTnI}$ and $\mathrm{cTnC}$, all three troponin proteins were detected even after washout for $30 \mathrm{~min}$ (Fig. 3e lane 3), corresponding to the force measurement shown in Fig. 3c. Taken together, these results indicate that the potentiation of contraction $(\sim 23 \%$ increase $)$, seen after the application of exogenous cTnI and cTnC, is due to construction of a Tn-complex-mediated contractile pathway, also reinforcing the working hypothesis that thin and thick filamentrelated pathways co-contribute to $\mathrm{Ca}^{2+}$-mediated smooth muscle contraction.

cTnT-deficient mice. To further confirm the role of cTnT in smooth muscle contraction, we next used $\mathrm{cTnT}^{+/-}$mice. The expression of cTnT in mRNA and protein was significantly reduced in urinary bladder detrusor smooth muscle of $\mathrm{cTnT}^{+/-}$mice (cTnT mRNA: $100 \pm 10.1 \%$ in WT mice, $\mathrm{n}=5$ vs $51.4 \pm 10.1 \%$ in $\mathrm{cTnT}^{+/-}$mice, $\mathrm{n}=5$ in; cTnT protein: $13.5 \pm 1.46 \times 10^{6}, \mathrm{n}=6$ vs $7.0 \pm 0.24 \times 10^{6}$ $\mathrm{n}=6$ ) (Fig. $4 \mathrm{a}-4 \mathrm{~d}$ ). $\left[\mathrm{Ca}^{2+}\right]_{\mathrm{i}}$-dependence of force development was examined in chemically permeabilized detrusor strips. $\left[\mathrm{Ca}^{2+}\right]$ of bathing solution increased cumulatively. The force development caused by $\mathrm{Ca}^{2+}<1 \mu \mathrm{M}$ was significantly smaller in $\mathrm{cTnT}^{+/-}$mice (at $0.3 \mu \mathrm{M}\left[\mathrm{Ca}^{2+}\right]_{\mathrm{i}}, 1.41 \pm 0.16 \mathrm{mg}$ vs $0.91 \pm 0.13 \mathrm{mg}, P=0.033$; at $0.5 \mu \mathrm{M}\left[\mathrm{Ca}^{2+}\right]_{\mathrm{i}}, 2.84 \pm 0.33 \mathrm{mg}$ vs $1.94 \pm 0.14 \mathrm{mg}, P=0.0017$, respectively; $\mathrm{n}=29$ in $\mathrm{WT}$ vs 34 in $\mathrm{cTnT}^{+/-}$mice), and it reached nearly the same level in both muscle strips at $\left[\mathrm{Ca}^{2+}\right]_{\mathrm{i}}>3 \mu \mathrm{M}$ (Fig. $4 \mathrm{e}$ ).
Muscarinic agonists such as acetylcholine (ACh) and carbachol (CCh), activate non-selective cation channels (NSCC) permeable to $\mathrm{Ca}^{2+}$ in detrusor smooth muscle ${ }^{19}$. In intact detrusor strips (without chemical permeabilization) from $\mathrm{WT}$ and $\mathrm{cTnT}^{+/-}$mice, the extracellular $\mathrm{Ca}^{2+}$ concentration $\left(\left[\mathrm{Ca}^{2+}\right]_{\mathrm{o}}\right.$ ) cumulatively increased in the presence of $1 \mu \mathrm{M} \mathrm{CCh}$ in order to alter $\left[\mathrm{Ca}^{2+}\right]_{\mathrm{i}}$ via NSCC (Fig. $4 \mathrm{f}$ ). The force development was again significantly smaller in $\mathrm{cTnT}^{+/-}$mice at lower extracellular $\mathrm{Ca}^{2+}$ concentrations $\left(P=0.024\right.$ at $0.03 \mathrm{mM}\left[\mathrm{Ca}^{2+}\right]_{\mathrm{o}}$ and $P$ $=0.041$ at $0.1 \mathrm{mM}\left[\mathrm{Ca}^{2+}\right]_{\mathrm{o}} ; \mathrm{n}=25$ in WT vs 30 in $\mathrm{cTnT}^{+/-}$mice). These results suggest that part of the contraction, especially medicated by a small rise in $\left[\mathrm{Ca}^{2+}\right]_{\mathrm{i}}$, utilizes the Tn complex and/or a Tn-like complex which includes replaceable proteins of Tn subunits (Fig. 1g).

\section{Discussion}

It is widely accepted that myosin thick-filaments make a major contribution to initiating smooth muscle contraction through phosphorylation of its light chain $^{3-5}$, on one hand, there is considerable evidence that thin filament-linked mechanisms are operating in parallel ${ }^{5-7}$. The present study has elucidated that cTnT plays a crucial role in the latter mechanism. Firstly, we have observed that cTnT is expressed in various smooth muscles (Fig. 1a and also see Fig. S4), and is the most abundant in the urinary bladder (Fig. 1c). A previous paper reports the expression of fast-twitch skeletal-type TnT (fTnT) in the murine aorta ${ }^{20}$. In contrast, our immunoblotting examinations have detected only cTnT in human detrusor smooth muscle, 
a

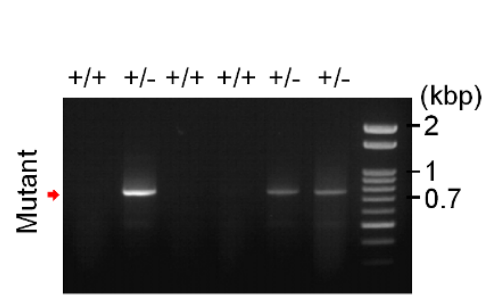

b

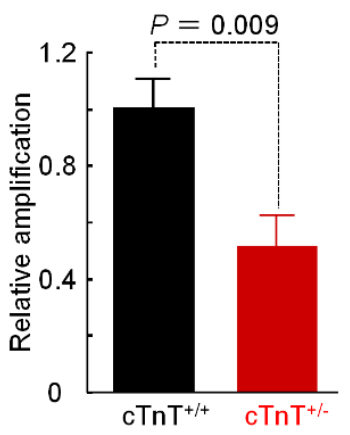

C

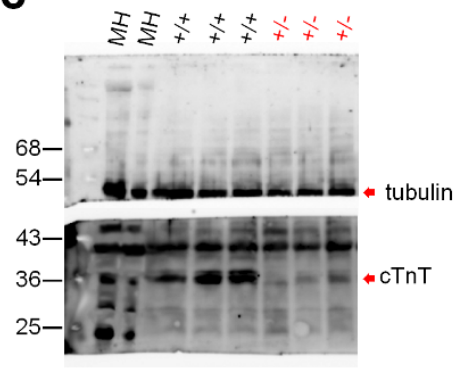

$\mathrm{MH}$ : mouse heart muscle d

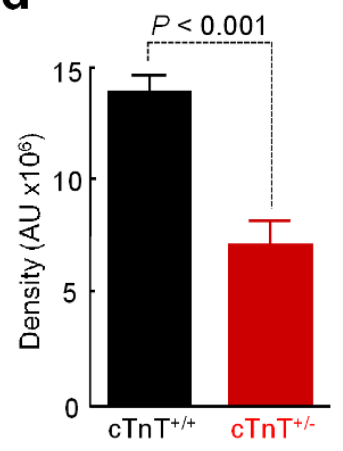

e
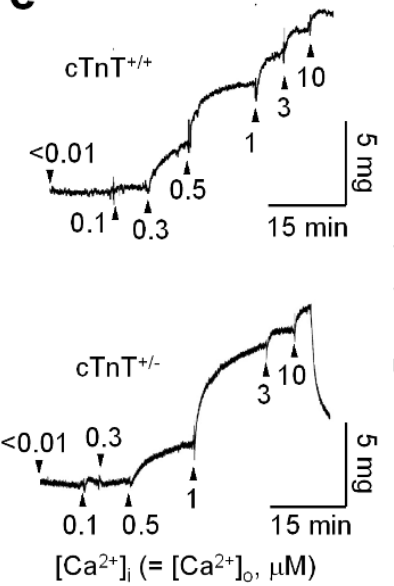

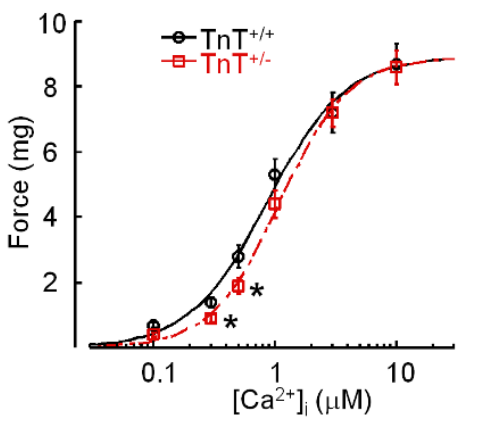

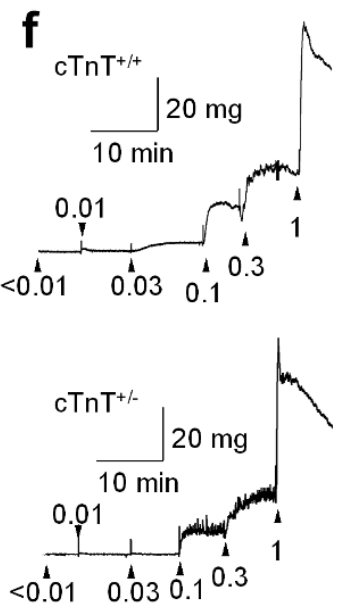

$\left[\mathrm{Ca}^{2+}\right]_{0}(\mathrm{mM})$

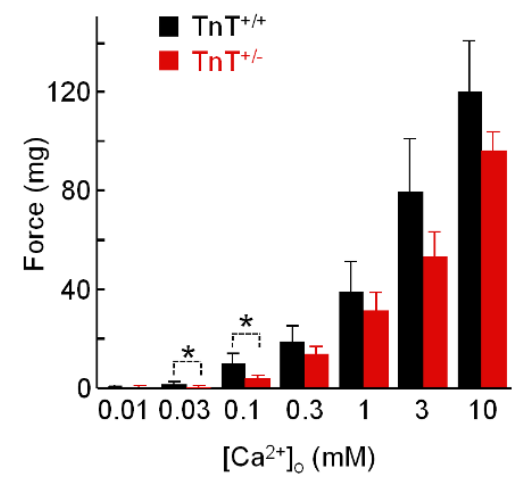

Figure $4 \mid$ Expression of cTnT and mechanical activity in WT $\left(\mathrm{cTnT}^{+/+}\right)$and $\mathrm{cTnT}^{+/-}$mice. (a) Genotyping of WT $\left(\mathrm{cTnT}^{+/+}\right)$and $\mathrm{cTnT}^{+/-} \mathrm{mice}$ Mutant bands at $0.76 \mathrm{kbp}$. (b) Real-time PCR quantification of cTnT. Expression of $\mathrm{cTnT}_{\mathrm{mRNA}}$ was normalized by the average value in $\mathrm{cTnT}^{+/+}$mice. ( $c$ and d) Western blot detection of cTnT is shown with $\beta$-tubulin in c. To quantify, cTnT content was normalized by $\beta$-tubulin in d. MH: Mouse heart. (e) Force development measured in chemically permeabilised strips of detrusor smooth muscle $(\sim 0.3 \times 0.3 \times 2 \mathrm{~mm})$ isolated from WT $\left(\mathrm{cTnT} \mathrm{T}^{+/+}\right)$and $\mathrm{cTnT}^{+/-}$mice ( $\mathrm{n}=28$ versus $34,{ }^{*} P<0.05$ : left panel). Force development $(F)$ is plotted against $\left[\mathrm{Ca}^{2+}\right]$ (right panel). The curves were drawn by fitting all data to the following equation: $F\left(\left[\mathrm{Ca}^{2+}\right]\right)=F_{\max } /\left\{1+\left(K_{\mathrm{M}} /\left[\mathrm{Ca}^{2+}\right]\right)^{H}\right\}$, where $K_{\mathrm{M}}$, and $H$ are $\left[\mathrm{Ca}^{2+}\right]$ for the half-maximal force development and Hill coefficient, respectively. The estimated $K_{\mathrm{M}}$ was $0.76 \mu \mathrm{M}$ in WT mice and $1.11 \mu \mathrm{M}$ in $\mathrm{cTnT}^{+/-}$mice. (f) Force development measured in intact detrusor strips in the presence of CCh (right panel), and the $\mathrm{Ca}^{2+}$-force relationship (left panel) in WT and $\mathrm{cTnT}^{+/-}$mice ( $\mathrm{n}=25 \mathrm{vs} 30$, unpaired $t$-test).

suggesting a wide variation of Tn expression among tissues and species. Furthermore, we have observed cTnT is colocalized with tropomyosin in the cytoplasm, implying that smooth muscle does not contain these proteins coincidently, but utilizes them in contraction via a thin-filament-linked signalling pathway. This finding also prompts us to consider possible detection of TnT-like proteins, for example calponin, which is known to be colocalized with tropomyosin in smooth muscle ${ }^{21,22}$. We have thus examined the sequences of the RT-PCR products, and confirmed that they are identical to the known cDNA sequences of Tns (Fig. S1).

Secondly, we have provided lines of functional evidence for the involvement of cTnT in smooth muscle contraction. In chemically permeabilized detrusor strips of humans, in which cTnT alone is detected in immunoblotting, application of both cTnI and cTnC causes significant potentiation of $\mathrm{Ca}^{2+}$-mediated contraction. Also, when cTnT content is genetically reduced (in $\mathrm{cTnT}^{+/-}$mice), the $\mathrm{Ca}^{2+}$-force relationship is shifted toward greater $\left[\mathrm{Ca}^{2+}\right]_{\mathrm{i}}$. In line with this observation, i.e. attenuation of $\left[\mathrm{Ca}^{2+}\right]_{i}$ sensitivity, muscarinic agonists induced contractions of smaller amplitude in intact detrusor strips of $\mathrm{cTnT}^{+/-}$mice at low extracellular $\mathrm{Ca}^{2+}$ concentrations $\left(\left[\mathrm{Ca}^{2+}\right]_{\mathrm{o}}\right)$. Together, these results indicate that the Tn complex and/or a Tn complex-like system involving TnT make a substantial contribution to smooth muscle contraction.
In smooth muscle, $\left[\mathrm{Ca}^{2+}\right]_{\mathrm{i}}$ is thought to be $\sim 100 \mathrm{nM}$ in basal conditions, and rises to $300 \mathrm{nM}-1.5 \mu \mathrm{M}$ in response to physiological stimuli including neurotransmitters and membrane depolarizations ${ }^{23,24}$. In this $\left[\mathrm{Ca}^{2+}\right]_{\mathrm{i}}$ range, the force development of chemically permeabilized smooth muscle preparations in $\mathrm{cTnT}^{+/-}$mice was $\sim 17-43 \%$ smaller than in WT mice (Fig. 4). cTnT is partially reduced (to $\sim 50 \%$ ) in $\mathrm{cTnT}^{+/-}$mice (Fig. 4B). Also, it is known that the TnT subtypes, i.e. cTnT/sTnT/fTnT are replaceable to form a Tn complex in reconstitution studies ${ }^{25}$. Therefore, in smooth muscle preparations of $\mathrm{cTnT}^{+/-}$ mice, skeletal muscle subtypes of $\mathrm{TnT}$, if any exists as for human smooth muscle, are likely to play a role. The actual contribution of thin-filament regulation via TnT to smooth muscle contraction is, thus, expected to exceed twice the apparent difference of the $\left[\mathrm{Ca}^{2+}\right]_{\mathrm{i}}$-tension relationship between $\mathrm{WT}$ and $\mathrm{cTnT}^{+/-}$mice.

Using permeabilized smooth muscle preparations, we have also successfully constructed the Tn system itself. Namely, the addition of exogenous cTnI followed by $\mathrm{cTnC}^{26}$ significantly potentiated the $\mathrm{Ca}^{2+}$-mediated force development (Fig. 3c). This implies that among Tn subunits, cTnT is predominant compared to cTnI and cTnC in smooth muscle, and that cTnT is already assembled with thin-filaments ready to convert the tropomyosin conformation, i.e. to cause thin-filament-linked contraction, when it receives the two other components of Tn. 
In striated muscles, the composition of Tn subunits and subtypes is known to alter during development and aging, and under pathological conditions ${ }^{27}$. Also, it is known that impaired RNA splicing and mutations of genes coding for Tns and related proteins such as tropomyosin, cause myopathies ${ }^{2,16}$. Such changes are likely to occur in numerous smooth muscles thereby altering contractility due to the $\mathrm{Tn} / \mathrm{Tn}$-like system. In addition, unidentified mutations of Tns, which mainly modulate smooth muscle contractility, may exist. Conversely, pharmacological and/or genetic treatments targeting the $\mathrm{Tn} / \mathrm{Tn}$-like system could be developed to overcome numerous diseases related to smooth muscle dysfunction, such as intractable hypertension, bronchial asthma, overactive bladder, intestinal hypomotility and so on. Furthermore, in the heart, some specific changes in the composition of Tn subtypes (expression of sTnI), affect the energetic cost of contraction and promote glycolysis ${ }^{28}$. Similar mechanisms might underlie characteristic features of smooth muscle contraction, such as the low energy cost, and the high rate of aerobic glycolysis ${ }^{29,30}$.

In conclusion, this study has elucidated the expression of Tns in smooth muscle and modulation of contractility via a $\mathrm{Tn} / \mathrm{Tn}$-like system, indicating that both thin and thick-filament-linked mechanisms are co-operating in $\mathrm{Ca}^{2+}$-mediated smooth muscle contraction (Fig. 1g). We assume that increases of $\left[\mathrm{Ca}^{2+}\right]_{i}$ in the physiological range (i.e. $300 \mathrm{nM} \sim 1.5 \mu \mathrm{M}$ ) preferably utilize the thin-filamentlinked mechanism including cTnT. On the other hand, the thickfilament-linked mechanism appears to make a major contribution to pharmacomechanical coupling, such as contractions induced by neurotransmitters and hormones which activate MLCK at low $\left[\mathrm{Ca}^{2+}\right]_{\mathrm{i}}$, and pathological contractions which increase $\left[\mathrm{Ca}^{2+}\right]_{\mathrm{i}}$ to extremely high concentrations. In order to develop new therapies, it merits exploring modulators of smooth muscle contractility via Tn/Tn-like systems.

\section{Methods}

Smooth muscle specimens. Detrusor smooth muscle specimens were obtained from humans and mice. Human detrusor was obtained from 7 males and 5 females with a mean age of $68.9 \pm 6.9$ years undergoing cystectomy due to bladder cancer. Voiding functions were almost normal. All patients provided informed written consent approved by the Kyushu University Hospital Ethical Committee.

Mice were treated ethically. All procedures were approved by the Institutional Animal Care and Use Committee. Wild-type $\left(\mathrm{WT}=\mathrm{cTnT}^{+/+}\right)$and $\mathrm{cTnT}^{+/-}$mice $(\sim 12$ weeks after birth) were sacrificed by cervical dislocation after deeply anaesthetising with diethyl ether, and the urinary bladder was quickly dissected. Since the homozygous cTnT gene knockout $\left(\mathrm{cTnT}^{-1-}\right)$ is lethal, heterozygous cTnT knockout $\left(\mathrm{cTnT}^{+/-}\right)$mice known to have a moderate decrease in $\mathrm{cTnT}$ expression despite having a normal life $\operatorname{span}^{31}$, were used in this study.

Genotyping was carried out 4 weeks after birth using total RNA (tRNA) extracted from the tail. PCR primers for the cTnT-deficient allele were: $(+) 5^{\prime}$ -

GATTGCACGCAGGTTCTCCG; (-) 5' -CAAGAAGGCGATAGAAGGCG. In Fig. $4 \mathrm{a}$, amplicons of $0.76-\mathrm{kbp}$ indicate the mutant band to distinguish WT and $\mathrm{cTnT}^{+/-}$mice in genotyping after birth.

Biochemical, immunohistochemical and physiological examinations were carried out using detrusor in which the urothelial mucosa and connective tissue were carefully removed under a binocular microscope.

mRNA expression. Total RNA (tRNA) was extracted with Torizol reagent (Invitrogen) from detrusor smooth muscle in the human urinary bladder. Also, human tRNA samples of several smooth muscle tissues (the trachea, aorta, stomach small intestine, colon, urinary bladder) and the heart were purchased from Clontech.

The expression of cTnT, cTnI, cTnC and GAPDH mRNAs were examined by reverse transcription-polymerase chain reaction (RT-PCR ${ }^{32}$. After treatment with RQ1 DNase (Promega), $1 \mu \mathrm{g}$ of the tRNA was subjected to a RT reaction in a $10 \mu \mathrm{L}$ reaction volume. RT was performed using random hexamer (12.5 pmol) and MMLV reverse transcriptase $(100 \mathrm{U})$, according to the manufacturer's instructions (Promega). Then, $1 \mu \mathrm{l}$ of RT product was used as a cDNA template for the PCR reaction. The resultant PCR amplicons were subcloned into pCR2.1 for TA cloning (Invitrogen) and DNA sequencing was carried out. The sequences of $\mathrm{cTnT}, \mathrm{cTnC}$ and cTnI amplicons are shown in Fig. S2, together with the primers used.

In commercial tRNA samples, and tRNA from detrusor smooth muscle of WT and $\mathrm{cTnT}^{+/-}$mice, mRNAs of Tn subunits were quantified using real-time PCR. tRNA $(2 \mu \mathrm{g})$ was subject to an RT reaction to produce $20 \mu \mathrm{l}$ of cDNA template. Then, the template was diluted $1: 50(\mathrm{v} / \mathrm{v})$, and $4.5 \mu \mathrm{l}$ of the diluted sample was mixed with $0.5 \mu \mathrm{l}$ TaqMan probe (in total $10 \mu \mathrm{l}$ reaction mixture). The TaqMan probes used were (ABI catalogue number): Hs02758991_g1 for human and mouse GAPDH;
Hs00165960_m1 (TNNT2) for human TnT type 2 (cardiac); Hs00165957_m1 (TNNI3) for human TnI type 3 (cardiac); Hs00268524_m1 (TNNC1) for human TnC type 1 (slow cardiac); Mm01290252_g1 (TNNT2) for mouse TnT type 2 (cardiac). The mRNA expression of cardiac troponins in each smooth muscle tissue was compensated by that of GAPDH.

Murine tRNA was also collected from several tissues, and the expression of cTnT was examined (Fig. S3).

Immunoblotting. Samples were separated by $12 \%$ SDS-polyacrylamide gel electrophoresis (SDS-PAGE) and transferred to a polyvinylidene difluoride membrane using a semi-dry transfer system (1 hr, $15 \mathrm{~V})$. After blocking with $5 \%$ skimmed milk for $1 \mathrm{hr}$, the membrane was probed with a first antibody. The membrane was washed three times and incubated with horseradish peroxidaseconjugated anti-mouse IgG (BioRad) for $1 \mathrm{hr}$. Immunoreactive proteins on the membrane were visualized by treatment with a detection reagent (LumiGLO, Cell Signaling Technology)

The monoclonal anti-cardiac TnT (cTnT) antibody was purchased from Hytest, the monoclonal anti-cardiac TnI (cTnI) antibody was from Fitzgerald, the monoclonal anti-slow muscle and-cardiac TnC (cTnC) antibody was from Abnova, while the monoclonal anti-tropomyosin antibody was from Sigma (St Louis, MO, USA).

Immunohistochemistry. Colocalization of TnT and tropomyosin was examined in thin sections (Fig. 2a, b). Formalin-fixed and paraffin-embedded detrusor smooth muscle was cut into small sections of $\sim 3 \mu \mathrm{m}$ thick. The sections were deparaffinized, dehydrated, and pretreated for heat-based antigen retrieval using $10 \mathrm{nM}$ citrate buffer solution ( $\mathrm{pH} \mathrm{6.0)}$ for $20 \mathrm{~min}$ in an autoclave. After blocking with $5 \%$ nonfat milk, the sections were incubated with monoclonal anti-cTnT antibody $(1: 100$ in PBS containing $5 \% \mathrm{BSA}$ and $0.1 \%$ Tween 20 ) overnight at $4{ }^{\circ} \mathrm{C}$. Inner peroxidase activity was blocked with methanol containing $1 \% \mathrm{H}_{2} \mathrm{O}_{2}$, and the sections were incubated with an HRP-labeled anti-mouse secondary antibody, HistoFine (DAKO, Glostrup, Denmark) for $30 \mathrm{~min}$. cTnT expression was visualized with the peroxidase stain DAB kit (Nacalai Tesque, Kyoto, Japan). Cell nuclei were counterstained with hematoxylin. Immunofluorescence staining was also performed. The sections treated with primary antibody were incubated with a biotinylated goat anti-mouse IgG secondary antibody ( $1: 200$; Vector Laboratories, Burlingame, CA). Thereafter, r-phycoerythrin (PE)conjugated streptavidin $(1: 100$; BD Biosciences, San Diego, CA) was applied, followed by 4,6-diamidino-2-phenylindole (DAPI) for counterstaining the nuclei. Immunofluorescence images were acquired using a fluorescence microscope (BZ8000, Keyence, Osaka, Japan).

Co-localization of cTnT and tropomyosin was also examined in cultured detrusor cells (Fig. 2c-1). Detrusor tissues obtained from patients were minced into small pieces and incubated for $1 \mathrm{~h}$ in Dulbecco's modified Eagle's medium (DMEM) (Sigma) containing $1 \mathrm{mg} / \mathrm{ml}$ collagenase type I (Worthington, Lakewood, NJ) and $10 \mathrm{U} / \mathrm{ml}$ elastase (Wako, Osaka, Japan). After digestion, isolated cells were washed with DMEM containing $10 \% \mathrm{FBS}, 100 \mu \mathrm{g} / \mathrm{ml}$ streptomycin, $100 \mathrm{units} / \mathrm{ml}$ penicillin and $50 \mu \mathrm{g} / \mathrm{ml}$ ascorbic acid, and kept in a $\mathrm{CO}_{2}$ incubator at $37^{\circ} \mathrm{C}$. Cultured cells of 6-10 passages were fixed in $4 \%$ formaldehyde and permeabilized in PBS containing $0.1 \%$ TritonX-100. Then cTnT and tropomyosin were labeled with their monoclonal antibodies (Hytest, Turku, Finland; Sigma St Louis, MO, USA) using a Zenon mouse IgG labeling kit (Invitrogen) according to the manufacturer's protocol. Cell nuclei were stained with DAPI. Negative controls of cTnT and tropomyosin were obtained from two types of human urinary bladder transitional cell carcinoma (UMUC3 and TCCSuP: left and right panels in Fig. S5, respectively). After permeabilization with Triton X-100, the same anti-cTnT and anti-tropomyosin antibodies were used to stain the cultured cells.

Force measurement. Small strips ( $\sim 0.5 \mathrm{~mm}$ wide and $1.0 \mathrm{~mm}$ long) of detrusor were prepared for force measurement. Small strips were initially mounted between two tungsten wires, one of which was fixed and the other was attached to a force transducer (UL2; Minebea, Osaka, Japan), at the centre of a Perspex block in a $60 \mu \mathrm{l}$ drop of physiological saline solution (PSS). The 'normal' physiological salt solution (PSS) had the following composition (mM): $\mathrm{NaCl} 138, \mathrm{KCl} 6, \mathrm{CaCl}_{2} 2.4$, glucose 12 and HEPES/Tris ( $\mathrm{pH}$ 7.3). Incubating solutions were changed by moving the Perspex block.

After stable responses had been achieved by exposure to $100 \mathrm{mM} \mathrm{K}^{+}$and/or $10 \mu \mathrm{M}$ Carbachol (CCh), detrusor strips were moved into relaxing solutions for a few minutes. Then, the cell membrane was permeabilised with $\beta$-escin ${ }^{33}$ with some modifications. The composition of the relaxing solution was $(\mathrm{mM})$ : potassium methansulphonate $100, \mathrm{Na}_{2} \mathrm{ATP} 2.2, \mathrm{MgCl}_{2} 3.38$, ethyleneglycol-bis ( $\beta$-aminoethylether)- $N^{\prime}, N^{\prime}, N^{\prime}, N^{\prime}$-tetra acetic acid (EGTA) 10 , creatine phosphate 10 , Tris-maleate 20 ( $\mathrm{pH}$ 7.2). The incubation time was $20-30 \mathrm{~min}$ for $20-40 \mu \mathrm{M} \beta$-escin. Activating solutions containing various ionized $\mathrm{Ca}^{2+}$ concentrations were made by adding appropriate amounts of $\mathrm{CaCl}_{2}$ to the relaxing solution, using the $\mathrm{Ca}^{2+}$-EGTA binding constant of $10^{6} / \mathrm{M}^{34}$. $\mathrm{Ca}^{2+}$-dependence of force development was examined at room temperature. The force became maximal at $\sim 10 \mu \mathrm{M}$, as previously reported in chemically permeabilised smooth muscles ${ }^{33,35}$.

To examine the effect of exogenous troponins, human cTnI and cTnC were cloned and expressed in Escherichia coli BL21(DE3). Protein purification was carried out as described previously ${ }^{36}$.

In some experiments, $\mathrm{Ca}^{2+}$-dependence of tension development was examined in intact detrusor strips (without permeabilization) in the presence of $\mathrm{CCh}^{37}$. The application of exogenous $\mathrm{cTnI}$ and $\mathrm{cTnC}$ had little effect in intact muscle preparations 
(Fig. S6). On the other hand, in chemically permeabilized detrusor strips, the application of $\mathrm{cTnI}$ alone suppressed $\mathrm{Ca}^{2+}$-mediated tension development (Fig. 3b), as previously observed ${ }^{38}$.

Statistics. Numerical data are expressed as mean \pm s.e. Significant differences were evaluated by two-tail, paired and unpaired $t$-tests. Single and double asterisks represent the probability $(P)<0.05$ and $<0.01$, respectively.

1. Ebashi, S. \& Endo, M. Calcium ion and muscle contraction. Prog. Biophys. Mol. Biol. 18, 123-183 (1968).

2. Ohtsuki, I. \& Morimoto, S. Troponin: regulatory function and disorders. Biochem. Biophys. Res. Commun. 369, 62-73 (2008).

3. Dillon, P. F., Aksoy, M. O., Driska, S. P. \& Murphy, R. A. Myosin phosphorylation and the cross-bridge cycle in arterial smooth muscle. Science 211, 495-497 (1981).

4. Ito, M. \& Hartshorne, D. J. Phosphorylation of myosin as a regulatory mechanism in smooth muscle. Prog. Clin. Biol. Res. 327, 57-72 (1990).

5. Kim, H. R., Appel, S., Vetterkind, S., Gangopadhyay, S. S. \& Morgan, K. G. Smooth muscle signalling pathways in health and disease. J. Cell. Mol. Med. 12, 2165-2180 (2008).

6. Smith, C. W., Pritchard, K. \& Marston, S. B. The mechanism of $\mathrm{Ca}^{2+}$ regulation of vascular smooth muscle thin filaments by caldesmon and calmodulin. J. Biol. Chem. 262, 116-122 (1987).

7. Kobayashi, H., Inoue, A., Mikawa, T., Kuwayama, H., Hotta, Y., Masaki, T. \& Ebashi, S. Isolation of cDNA for bovine stomach $155 \mathrm{kDa}$ protein exhibiting myosin light chain kinase activity. J. Biochem. 112, 786-789 (1992).

8. Ansari, S., Alahyan, M., Marston, S. B. \& El-Mezgueldi, M. Role of caldesmon in the $\mathrm{Ca}^{2+}$ regulation of smooth muscle thin filaments: evidence for a cooperative switching mechanism. J. Biol. Chem. 283, 47-56 (2008).

9. Jin, J. P., Zhang, Z. \& Bautista, J. A. Isoform diversity, regulation and functional adaptations of troponin and calponin. Crit. Rev. Eukar. Gene Expr. 18, 93-124 (2008).

10. Rozenblum, G. T. \& Gimona, M. Calponins: adaptable modular regulators of the actin cytoskeleton. Int. J. Biochem. Cell Biol. 40, 1990-1995 (2008).

11. Chatterjee, M. \& Tejada, M. Phorbol ester-induced contraction in chemically skinned vascular smooth muscle. Am. J. Physiol. 251, C356-361 (1986).

12. Fulginiti, 3rd J., Singer, H. A. \& Moreland, R. S. Phorbol ester-induced contractions of swine carotid artery are supported by slowly cycling crossbridges which are not dependent on calcium or myosin light chain phosphorylation. J. Vasc. Res. 30, 315-322 (1993).

13. Walsh, M. P., Andrea, J. E., Allen, B. G., Clément-Chomienne, O., Collins, E. M. \& Morgan, K. G. Smooth muscle protein kinase C. Can. J. Physiol. Pharmacol. 72 , 1392-1399 (1994).

14. Su, X., Smolock, E. M., Marcel, K. N. \& Moreland, R. S. Phosphatidylinositol 3-kinase modulates vascular smooth muscle contraction by calcium and myosin light chain phosphorylation-independent and -dependent pathways. Am. J. Physiol. Heart Circ. Physiol. 286, H657-666 (2004).

15. Scruggs, S. B. \& Solaro, R. J. The significance of regulatory light chain phosphorylation in cardiac physiology. Arch. Biochem. Biophys. 510, 129-134 (2011).

16. Ochala, J. Thin filament proteins mutations associated with skeletal myopathies: defective regulation of muscle contraction. J. Mol. Med. (Berl) 86, 1197-1204 (2008)

17. Ngai, P. K., Scott-Woo, G. C., Lim, M. S., Sutherland, C. \& Walsh, M. P. Activation of smooth muscle myosin $\mathrm{Mg}^{2+}$-ATPase by native thin filaments and actin/ tropomyosin. J. Biol. Chem. 262, 5352-5359 (1987).

18. Smillie, L. B. Tropomyosin. In Biochemistry of smooth muscle contraction. M. Bárány, ed. (San Diego, California: Academic Press), pp. 63-75 (1996).

19. Kajioka, S., Nakayama, S., Asano, H. \& Brading, A. F. Involvement of ryanodine receptors in muscarinic-receptor-mediated membrane current oscillation in urinary bladder smooth muscle. Am. J. Physiol. Cell Physiol. 288, C100-108 (2005).

20. Moran, C. M., Garriock, R. J., Miller, M. K., Heimark, R. L., Gregorio, C. C. \& Krieg, P. A. Expression of the fast twitch troponin complex, fTnT, fTnI and fTnC, in vascular smooth muscle. Cell Motil. Cytoskeleton 65, 652-661 (2008).

21. Takahashi, K., Hiwada, K. \& Kokubu, T. Vascular smooth muscle calponin. A novel troponin T-like protein. Hypertension 11, 620-626 (1988).

22. Walsh, M. P., Carmichael, J. D. \& Kargacin, G. J. Characterization and confocal imaging of calponin in gastrointestinal smooth muscle. Am. J. Physiol. 265, C1371-1378 (1993).

23. Karaki, H., Ozaki, H., Hori, M., Mitsui-Saito, M., Amano, K., Harada, K., Miyamoto, S., Nakazawa, H., Won, K. J. \& Sato, K. Calcium movements, distribution, and functions in smooth muscle. Pharmacol. Rev. 49, 157-230 (1997).
24. Wray, S., Burdyga, T. \& Noble, K. Calcium signalling in smooth muscle. Cell Calcium 38, 397-407 (2005).

25. Liou, Y. M. \& Chang, J. C. Differential pH effect on calcium-induced conformational changes of cardiac troponin $\mathrm{C}$ complexed with cardiac and fast skeletal isoforms of troponin I and troponin T. J. Biochem. 136, 683-692 (2004).

26. Rüegg, J. C., Zeugner, C., van Erk, J., Kay, C. M. \& Hodges, R. S. Inhibition of TnITnC interaction and contraction of skinned muscle fibres by the synthetic peptide TnI. Pflügers Arch. 414, 430-436 (1989).

27. Anderson, P. A., Greig, A., Mark, T. M., Malouf, N. N., Oakeley, A. E., Ungerleider, R. M., Allen, P. D. \& Kay, B. K. Molecular basis of human cardiac troponin T isoforms expressed in the developing, adult, and failing heart. Circ. Res. 76, 681-686 (1995).

28. Pound, K. M., Arteaga, G. M., Fasano, M., Wilder, T., Fischer, S. K., Warren, C. M., Wende, A. R., Farjah, M., Abel, E. D., Solaro, R. J. \& Lewandowski, E. D. Expression of slow skeletal TnI in adult mouse hearts confers metabolic protection to ischemia. J. Mol. Cell. Cardiol. 51, 236-243 (2011).

29. Clark, J. F., Kemp, G. J. \& Radda, G. K. The creatine kinase equilibrium, free [ADP] and myosin ATPase in vascular smooth muscle cross-bridges. J. Theor. Biol. 173, 207-211 (1995).

30. Lynch, R. M. \& Paul, R. J. Compartmentation of carbohydrate metabolism in vascular smooth muscle. Am. J. Physiol. 252, C328-334 (1987).

31. Nishii, K., Morimoto, S., Minakami, R., Miyano, Y., Hashizume, K., Ohta, M., Zhan, D. Y., Lu, Q. W. \& Shibata, Y. Targeted disruption of the cardiac troponin T gene causes sarcomere disassembly and defects in heartbeat within the early mouse embryo. Dev. Biol. 322, 65-73 (2008).

32. Kajioka, S., Nakayama, S., Asano, H., Seki, N., Naito, S. \& Brading, A. F. Levcromakalim and GDP activate small conductance $\mathrm{K}_{\mathrm{ATP}}$ channels with the composition of Kir6.1/SUR2A in pig detrusor smooth muscle cells: Uncoupling of cAMP signal pathways. J. Pharmacol. Exp. Ther. 327, 114-123 (2008).

33. Durlu-Kandilci, N. T. \& Brading, A. F. Involvement of Rho kinase and protein kinase $\mathrm{C}$ in carbachol-induced calcium sensitization in beta-escin skinned rat and guinea-pig bladders. Br. J. Pharmacol. 148, 376-384 (2006).

34. Saida, K. \& Nonomura, Y. Characteristics of $\mathrm{Ca}^{2+}$ - and $\mathrm{Mg}^{2+}$-induced tension development in chemically skinned smooth muscle fibers. J. Gen. Physiol. 72, 1-14 (1978).

35. Iino, M. Tension responses of chemically skinned fibre bundles of the guinea-pig taenia caeci under varied ionic environments. J. Physiol. 320, 449-467 (1981)

36. Yanaga, F., Morimoto, S. \& Ohtsuki, I. $\mathrm{Ca}^{2+}$ sensitization and potentiation of the maximum level of myofibrillar ATPase activity caused by mutations of troponin T found in familial hypertrophic cardiomyopathy. J. Biol. Chem. 274, 8806-8812 (1999).

37. Baba, K., Satake, T., Takagi, K. \& Tomita, T. Effects of verapamil on the response of the guinea-pig tracheal muscle to carbachol. Br. J. Pharmacol. 88, 441-449 (1986).

38. Watanabe, M., Yoshino, Y. \& Morimoto, S. Troponin I inhibitory peptide suppresses the force generation in smooth muscle by directly interfering with cross-bridge formation. Biochem. Biophys. Res. Commun. 307, 236-240 (2003).

\section{Acknowledgements}

The authors are grateful to Professor Joseph F. Clark (University of Cincinnati, USA) for stimulating discussion. This work was partly supported by Grant-in-Aid for Scientific Research from the Japan Society for the Promotion Science.

\section{Author contributions}

S.K., N.S., H.M., S.M., N.S. and T.S. performed experiments on force development, and analysed the data. F.T.-Y., M.M. and H.A. performed PCR and western blot. M.O. and Y.Y. contributed to immunohistochemistry. S.N. and S.K. designed experiments, prepared smooth muscle specimens and performed PCR. S.N., S.K., S.Naito and M.H. wrote the manuscript.

\section{Additional information}

Supplementary information accompanies this paper at http://www.nature.com/ scientificreports

Competing financial interests: The authors declare no competing financial interests.

License: This work is licensed under a Creative Commons

Attribution-NonCommercial-NoDerivs 3.0 Unported License. To view a copy of this license, visit http://creativecommons.org/licenses/by-nc-nd/3.0/

How to cite this article: Kajioka, S. et al. Endogenous Cardiac Troponin T Modulates $\mathrm{Ca}^{2+}$-Mediated Smooth Muscle Contraction. Sci. Rep. 2, 979; DOI:10.1038/srep00979 (2012). 